\title{
Postural thixotropy: a significant factor in the stiffness of paralysed limbs?
}

\author{
E G Walsh MD FRCP FRSE
}

Department of Physiology, University of Edinburgh, Edinburgh EH8 9AG, Scotland.

The term 'thixotropy' is not at present widely used in the medical literature. It was introduced in 1927 by Péterfi who stirred the cytoplasm of sea urchin eggs with a needle and noticed a change from a gel to a sol; instead of being stiff there developed the consistency of a fluid. On being left undisturbed for some time the sol again became a gel. Thus, at it's origin the term had to do with a stiffness which was reduced by motion, and the stiffness took some time to be re-established; the material had a 'memory'. The etymology is an origin from 2 Greek words ' $\theta \iota \xi \iota s$ ', thixis, touch and ' $\tau \rho \circ \pi$ os', tropos, state.

For about 20 years I have sought to investigate problems concerning the measurement and regulation of muscle tone in normal people and in patients with a wide range of neurological abnormalities. The studies have included, in recent years, some measurements of tone in the muscles around the knee in paraplegic patients. The material has been published in a journal devoted to physiology. ${ }^{1,2}$ I wish now to discuss a possible factor which may be significant and which has not, I think, been approached in other laboratories. Where necessary appropriate informed consent and ethical permission were obtained.

My initial studies were concerned with the wrists of normal people. Rhythmic forces were applied from a special motor. It was found that at a certain frequency the oscillation was greatest. This 'resonant frequency' reflected the stiffness of the muscles and it was observed to be higher, indicating greater stiffness, for small as compared with larger movements. This was both a puzzle and a lead. ${ }^{3}$

Observations of a similar type were made on patients who, for surgical operations, were anaesthetised and treated with full doses of a neuromuscular blocking agent. The stiffening with small forces was again seen, indicating that it was a passive property of the tissues and was not due to some special neural control mechanism.

In other observations the wrist was oscillated with small rhythmic forces at about $3 \mathrm{~Hz}$. At a certain level of force a brief passive disturbance loosened up the system, and the motion thereafter, for the same force, was increased to double or more. The loosened state persisted as long as the force was applied. If however the force was cut out for a period and then reapplied the motion was reduced to about the original level.

Observations were made at a number of other joints of the body. In each example similar phenomena were found.

Stiffness which is history dependent is characteristic of thixotropy. There are sol/ gel tranformations, of solutions of long chained molecules. The change from a gel to a sol is induced by motion; left undisturbed the system reverts to the gel state and stiffens up. Clays and some paints are thixotropic and bentonite is added to calamine lotion because of its thixotropic properties. If the bottle is shaken the lotion can be applied as a liquid, left to itself on the skin it stays in place as a paste.

Thixotropy has been shown to occur in isolated muscle. ${ }^{4}$ It may be a function of the cross-bridges between the actin and myosin filaments which can be separated by a moderately large movement.

The stiffening with immobility occurs in 2 phases. There is a very rapid change in the first few seconds of rest followed by further stiffening having a much slower time course. $^{5}$

Observations were made on subjects who had the forearm cooled whilst the thixotropic effects were observed at the wrist. With cooling loosening up did not occur or was greatly reduced. ${ }^{6}$

Paralysed limbs are immobile and often 
cold. Both factors suggest that a high level of thixotropic stiffening will occur. I have not had, or made, the opportunity of making extensive observations on this phenomenon in patients with spinal injuries but report here observations on a tetraplegic patient with a complete lesion at C7.

The arrangements are shown in Figure 1. The patient lay on his side and a printed motor concentric with the knee was coupled to the leg by a light alloy splint and bandage. The motion was recorded by a low friction potentiometer concentric with the axis of the motor.

One result is shown in Figure 2. There was some low level ongoing activity in the EMG of the quadriceps, whilst the hamstrings were silent. It will be seen that a rhythmic torque initially produced a little oscillation. The leg was then displaced by hand to generate a single bigger movement. Thereafter the motion was clearly greater. Another similar record is shown in Figure 3.

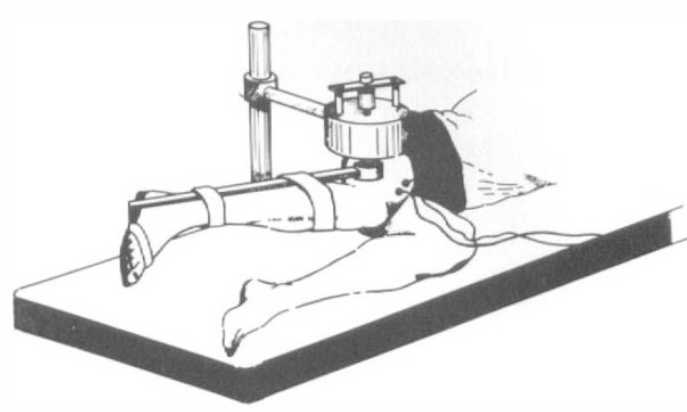

Figure 1 The cylindrical printed motor is above and concentric with the knee. It is controlled by a power amplifier in the current feedback mode so that the input voltage from a waveform generator provides a corresponding current through the disc-like armature. ${ }^{1}$

The motion after the disturbance was increased several fold.

A number of the procedures used by physiotherapists will loosen thixotropic stiffening. Thus passive and active motion, heat

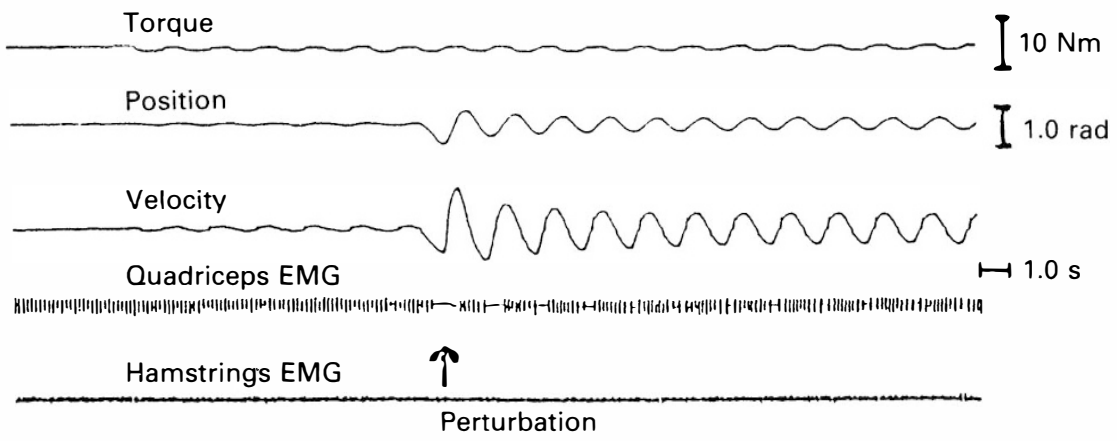

Figure 2 The motor generates an oscillatory torque at about $1 \mathrm{~Hz}$. The oscillations of the paralysed leg are greatly increased after the perturbation produced by moving the limb by hand.

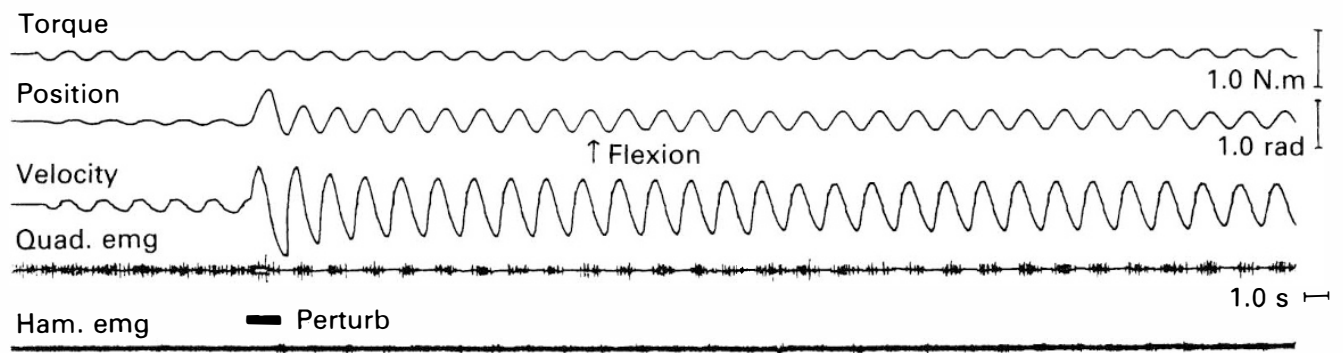

Right knee

Figure 3 Another example of loosening by a moderately large motion at the knee of the tetraplegic patient. 
and diathermy and perhaps ultrasonics may all disrupt the bonds which have formed. It would be important to document these changes more fully, with a view to improved quality control of the efficacy of different therapeutic manoeuvres.

\section{References}

1 Douglas AJ, Walsh EG, Wright WG, Edmond P (1989) Muscle tone around the human knee in paraplegia. Q J Exp Physiol 74: 897-905.

2 Douglas AJ, Walsh EG, Wright GW, Creasey GH, Edmond P (1991) The effects of neuromuscular stimulation on muscle tone at the knee in paraplegia. Exp Physiol 76: 357-367.

3 Lakie M, Walsh EG, Wright GW (1984) Resonance at the wrist demonstrated by the use of a torque motor: an instrumental analysis of muscle tone in man. $J$ Physiol 353: 265-285.

4 Lakie M, Robson LG (1988) Thixotropy: the effect of stretch size in relaxed frog muscle. Q J Exp Physiol 73: $127-129$.

5 Lakie M, Robson LG (1988) Thixotropic changes in human muscle stiffness and the effects of fatigue. $Q J$ Exp Phys 73: 487-500.

6 Lakie M, Walsh EG, Wright GW (1986) Control and postural thixotropy of the forearm muscles: changes caused by cold. J Neurol Neurosurg Psychiatry 49: 69-76. 